\title{
Hypothermic Circulatory Arrest Time affects neurological outcomes of Frozen Elephant Trunk for Acute Type A Aortic Dissection: a systematic review and meta-analysis
}

\author{
Mostafa Mousavizadeh ${ }^{1}$, Mahdi Daliri ${ }^{1}$, Hadi Abo Aljadayel ${ }^{1}$, Mohammed Idhrees ${ }^{2}$, Yousef \\ Rezaei $^{1}$, Mohamad Bashir ${ }^{3}$, and Saeid Hosseini ${ }^{1}$ \\ ${ }^{1}$ Rajaie Cardiovascular Medical and Research Center \\ ${ }^{2}$ SRM Institutes for Medical Science Vadapalani \\ ${ }^{3}$ Royal Blackburn Hospital
}

April 27, 2021

\begin{abstract}
Background: The treatment of complex thoracic aorta pathologies remains a challenge for cardiovascular surgeons. After introducing Frozen Elephant Trunk (FET), a significant evolution of surgical techniques has been achieved. The present metaanalysis aimed to assess the efficacy of FET in acute type A aortic dissection (ATAAD) and the effect of circulatory arrest time on post-operative neurologic outcomes. Methods: A standard Preferred Reporting Items for Systematic Reviews and Meta-Analyses search was conducted for all observational studies of patients diagnosed with ATAAD undergoing total arch replacement with FET reporting in-hospital mortality, bleeding, and neurological outcomes. A random-effect meta-analysis was performed using STATA software (StataCorp, TX, USA). Results: Thirty-five studies were eligible for the present meta-analysis, including 3211 patients with ATAAD who underwent total arch replacement with FET. The pooled estimate for in-hospital mortality, postoperative stroke, and spinal cord injury were 7\% (95\% CI 5-9; I2 =68.65\%), 5\% (95\% CI $4-7 ; \mathrm{I} 2=63.93 \%$ ), and $3 \%(95 \%$ CI $2-4 ; \mathrm{I} 2=19.56 \%)$, respectively. Univariate meta-regression revealed that with increasing the duration of hypothermic circulatory arrest time, the effect sizes for postoperative stroke and SCI enhances. Conclusions: It seems that employing the FET procedure for acute type A dissection is associated with acceptable neurologic outcomes and a similar mortality rate comparing with other aorta pathologies. Besides, increasing hypothermic circulation arrest time appears to be a significant predictor of adverse neurologic outcomes after FET.
\end{abstract}

\section{Introduction}

Utilizing "elephant trunk" (ET) prosthesis first described by Borst et al. in 1986 for aneurysmal aortic disease revolutionized treatment strategies in managing patients with complex arch and descending aorta pathologies (1). Antegrade open stent-grafting of the descending aorta described in 1996 became the cornerstone of further advancement in the arch and proximal descending aorta (2). In the early 2000s, a custom-made hybrid prosthesis called frozen elephant trunk (FET) had been developed (3). Subsequently, the FET technique has been extensively engaged in various acute and chronic pathologies of the thoracic aorta.

Replacement of the ascending aorta is a well-researched conventional treatment of acute Type A aortic dissection (ATAAD); however, residual pathology or dissection in the arch or downstream descending aorta may require additional treatment with high-risk redo procedures. On the other hand, more extensive aorta replacement (e.g., employing FET) carries the risk of complications. Therefore, there is still an ongoing debate over the optimal indication and FET use in ATAAD. One of the main complications associated with FET is neurologic events, particularly spinal cord injury (SCI) and stroke. The incidence rate of permanent 
or transient ischemic SCI after conventional ET has been reported between $0.4 \%$ and $2.8 \%$ in previous reports (4).

In contrast, the incidence of SCI after FET ranged between $8 \%$ in multicenter studies (5) and $20 \%$ in singlecenter reports (6). Several contributors (i.e., length of the device and distal circulatory arrest time) have been proposed to explain SCI and stroke observed after FET procedure, mainly derived from observational studies on patients with diverse aortic pathologies. Previous meta-analyses performed on primary studies included a mixture of cohorts diagnosed with acute or chronic dissection which differ in various aspect of treatment options and also comorbid diseases (7). Herein, we evaluated the pre-and intra-operative characteristics of patients with ATAAD undergoing the FET procedure and their associations with postoperative outcomes.

\section{Methods}

\section{Literature Search and design}

The review team developed the literature search strategy. Studies related to the subject were identified by searching electronic databases and bibliographic reference lists of relevant articles. An electronic search in PubMed/Medline, EMBASE, Scopus, and Web of Science databases was conducted from their beginning to June 2020 to identify studies reporting the outcomes of FET procedure in cohorts of patients diagnosed with ATAAD. A combination of controlled vocabulary and free text terms (utilizing Boolean operators) was used to interrogate the databases. The search was limited to English-language articles and human studies. The search strategy is presented in supplementary table 1. The present study is conducted and reported following the Preferred Reporting Items for Systematic reviews and Meta-Analyses (PRISMA) guidelines (8).

\section{Eligibility Criteria of Studies and Data Extraction}

Studies ascertained eligibility if those provided total aortic arch replacement with FET through median sternotomy in the ATAAD patients. Proximal arch or hemiarch procedures (i.e., procedures that did not replace the entire arch) and other pathologies (i.e., aneurysm) were excluded. Additional inclusion criteria included all observational studies reporting more than 20 patients undergoing the FET procedure and providing data for postoperative outcomes. Studies reported a combination of patients with varying pathologies was excluded if pre- and post-operative data were not reported for ATAAD individually. To exclude duplicated or overlapped populations from the same institution, these publications were identified. The latest report or those with the largest sample size without overlapped time were included in the final analysis.

After excluding case reports, conference presentations, editorials, expert opinions, and duplicated evidence, all titles and abstracts were reviewed by two independent reviewers (MM and YR), and irrelevant ones were excluded. The full-text articles were retrieved in those that met our inclusion criteria (Figure 1). The data extraction was independently performed by two authors (MM and YR). If necessary, discrepancies were resolved by consensus or discussion with the senior and corresponding authors (MB and $\mathrm{SH}$ ). The extracted data included baseline characteristics (i.e., age, sex, cardiovascular risk factors, smoking history, prior surgery, chronic kidney disease, and history of stroke). Procedure-related features (i.e., concomitant procedures, procedure duration, cardiopulmonary bypass time, circulatory arrest time, cannulation site, and stent type) and postoperative follow-up outcomes (i.e. mortality, acute kidney injury, post-operative bleeding, transient or permanent stroke, and spinal cord injury) were also extracted and recorded.

\section{Statistical analysis}

Study features and patients' characteristics were reported. Data are presented as number (percentage) or mean and $95 \%$ confidence interval (CI), as appropriate. The pooled estimated prevalence of each outcome was calculated utilizing a random-effect model, which is more generalized when potential heterogeneity among studies is present. All values were presented with $95 \%$ confidence intervals (CI), and the weighted prevalence of outcomes is depicted as a forest plot. We assessed the homogeneity of incidence of events across studies using Cochran Q for each of the outcomes. Also, to test for heterogeneity, Higgins $I^{2}$ was calculated. $I^{2}$ represents the percentage of total variation across studies that can be attributed to heterogeneity rather than 
chance. The $I^{2}$ value $0 \%$ indicates no heterogeneity, $25 \%$ low heterogeneity, $50 \%$ moderate heterogeneity, and $75 \%$ high heterogeneity (9). Funnel plots were drawn for all extracted outcomes to assess publication bias. Visual inspection of asymmetry was assessed to check for publication bias, and Egger's test was also conducted to evaluate small-study effects on the pooled estimated outcome (10). Moreover, Moment base univariate meta-regression was performed to assess the effects of hypothermic circulatory arrest (HCA) time on neurologic outcomes (stroke and SCI), depicted as bubble plot. All data were analyzed using STATA software (StataCorp, TX, USA).

\section{Results}

\section{Baseline characteristics and procedural features}

After reviewing shreds of evidence, a total of 35 studies including 3211 patients were entered into the quantitative analysis (Figure 1)(11-44). The median size of reviewed reports was 72 patients (interquartile range $38-122$ ). The majority of studies were performed in Chinese institutions, 19 studies including 2026 patients. The patients' median age was 52 years (interquartile range $48-59$ ). The frequency of male and female genders were $2268(74.6 \%)$ and $773(25.4 \%)$, respectively, reporting by 34 studies. Smoking (208 patients, 43.1\%) and hypertension (1827 patients, 70\%) were reported by seven and 29 studies. Other baseline characteristics are summarized in Table 1 and Supplementary Table 2.

Emergent/urgent surgery was implemented in 952 patients, reported by 11 studies. The cannulation site was applied in the innominate artery and the axillary artery in 3 studies (245 patients) and 20 studies (1896 patients), respectively. Bentall and David procedures were performed in 566 of 2152 patients (22 studies) and 219 of 1550 patients (14 studies). The ascending aorta replacement was applied in 364 patients, reporting by six studies. The type of stent deployed in the descending aorta included Cronus (MicroPort Medical, Shanghai, China), E-vita (JOTEC GmbH, Hechingen, Germany), Thoraflex (Vascutek, Inchinnan, UK), and Frozenix (Japan Lifeline, Tokyo, Japan) that those were implanted in 1413, 315, 250, and 161 patients, respectively. The stent type in other patients is also provided in Table 2. Only 342 patients in four studies reported the use of near-infrared spectroscopy during surgery. The median of HCA time was 31 min (interquartile range $25-35$ ), reported by 19 studies. Other surgery-related features are summarized in Table 2 .

\section{Follow-up outcomes}

The pooled estimates of postoperative stroke and SCI were $5 \%\left(95 \%\right.$ CI $\left.4-7 ; I^{2}=63.93 \%\right)$ and $3 \%(95 \%$ CI $2-4 ; I^{2}=19.56 \%$ ), respectively (Figure 2 ). The pooled estimates of acute kidney injury, bleeding, and in-hospital mortality were found to be $12 \%$ (95\% CI $\left.9-15 ; I^{2}=86.65 \%\right), 6 \%$ (95\% CI $3-8 ; I^{2}=71.05 \%$ ), and $7 \%\left(95 \%\right.$ CI $5-9 ; I^{2}=68.65 \%$ ), respectively (Figure 3 ).

\section{Publication bias and meta-regression}

The visual inspection of Funnel plots revealed asymmetries among studies reporting major postoperative outcomes (Supplemental Figure 1). Based on the Egger's test, there were significant for all major outcomes, including postoperative stroke $(\mathrm{p}<0.0001)$, SCI $(\mathrm{p}=0.004)$, acute kidney injury $(\mathrm{p}<0.0001)$, bleeding $(\mathrm{p}<0.0001)$, and in-hospital mortality $(\mathrm{p}<0.0001)$. The univariate meta-regression showed significant relationships between the HCA time and the development of postoperative stroke $(\mathrm{p}=0.04)$ and SCI $(\mathrm{p}=$ 0.05). Also, the bubble plots revealed that with increasing HCA time, the effect sizes for postoperative stroke and SCI enhance. The regression line provides a good fit for the data because most studies are relatively close (Figure 4).

\section{Discussion}

Nowadays, the most common indication for FET is chronic or acute dissection that involved the ascending aorta followed by degenerative atherosclerosis aneurysm (45). The FET procedure is now potentially indicated for varying aortic arch and descending aorta pathologies, including chronic or acute dissection as well as aneurysmal pathologies. It is well established that each pathology is associated with specific risk profiles, 
affecting short- and long-term outcomes after aortic arch repair (46). In addition to the type of aorta pathology, cannulation site, concomitant surgery, neurological monitoring, the method surgeon approached to the left subclavian artery, and intra-operative measures would change the course of postoperative outcomes. So, we herein conducted a systematic review focused on the outcome of FET deployment in ATAAD patients who needs total arch replacement, taking into account peri-operative features.

The present meta-analysis, including 35 studies with 3211 patients diagnosed with ATAAD, revealed that the FET procedure in this subset of patients was associated with $3 \%(95 \%$ CI $2-4)$ and $5 \%$ (95\% CI $4-7)$ of postoperative SCI and stroke, respectively. Additionally, the pooled rate of in-hospital mortality was found to be $7 \%$. A recent meta-analysis by Preventza et al. demonstrated a total pooled operative mortality of $8.8 \%$ from 34 reports on patients undergoing FET procedure (7) comparable with rates reported by other metaanalyses $(47,48)$. In the present meta-analysis, we found that the pooled estimate of in-hospital mortality was $7 \%\left(95 \%\right.$ CI $\left.5-9 ; I^{2}=68.65 \%\right)$ which is less than previous reports. One possible explanation is that they estimated operative mortality (variously reported operative, 30-day, and in-hospital), not specifically in-hospital mortality estimated in our analysis. Another drawback regarding previous reports is that they included heterogenous populations with different pathologies or chronicity of dissection in the final analysis. Although they tried to address this issue by subgroup analysis (7), only 12 studies on patients with ATAAD have been compared with other pathologies (14 studies). Besides, recent meta-analyses $(47,48)$ included a mixture of patients who underwent total arch replacement, proximal, and hemi arch replacement; meanwhile, they reported a higher mortality rate. It is noteworthy that total arch replacement is a more complex surgery. Hence, total arch replacement in an urgent or emergent situation inheres with a greater perioperative mortality rate. The observed discrepancy and declining mortality rate during recent years may be attributed to improved diagnostic modalities, enhanced experience of aortic centers and aortic surgeons, and well-established monitoring techniques (49).

Despite the improved results of extensive aortic surgery since the introduction of HCA and brain protection techniques, these procedures are still associated with neurologic impairments. Neurologic complications such as stroke and SCI are the most disastrous complication of aortic surgery, especially after thoracic and arch procedures, with a high burden of morbidity and health-related costs (50). In a single-center series of 25 patients who underwent thoracic aorta aneurysm repair with FET, $24 \%$ of patients developed SCI after surgery which is a significant rate of this dreaded complication (6). On the other hand, data from multicenter studies revealed a considerably lower rate of stroke and SCI. A report from the International E-vita Open Registry (IEOR) indicated a $6 \%$ and $8 \%$ incidence rate of stroke and SCI, respectively (5). We found that the pooled estimate for overall stroke reported in 24 studies was $5 \%$, and $3 \%$ for SCI reported in 16 studies. A different definition of SCI and diagnostic criteria and distinct baseline characteristics could explain the inconsistency observed amongst previous reports. However, the impact of the indication of total arch replacement (ATAAD versus chronic dissection and aneurysm) is arguable. This was the main rationale behind including only studies of patients diagnosed with ATAAD in the present meta-analysis. Several hypotheses proposed to explain SCI after FET, including the level of distal landing zone of the stent, hemodynamic instability after cardiopulmonary bypass, age, compromising segmental spinal cord blood supply, and HCA time $(6,51,52)$. The effect of the distal stent landing zone on the incidence of SCI has been explored previously; however, different results indicated the possibility of other contributing factors $(6,53)$. We examined the effect of HCA time on the development of neurologic adverse events. Nowadays, isolated HCA as a protective strategy is not recommended (36). Recent publications indicate that milder hypothermic circulatory arrest in combinations of routine selective antegrade cerebral perfusion improves brain and end-organ protection (54). Unfortunately, we could not evaluate the relationship between core temperature during arch replacement and neurologic events due to lack of data and inconsistency between reports regarding measurements. A total of 19 studies reported circulatory arrest time, and we observed that with a longer duration of circulatory arrest time, a higher rate of neurologic adverse events would be expected. A similar concern regarding the role of HCA time has been raised by Tian et al. while they found a significant strong positive linear relationship between mortality and circulatory arrest time $(\mathrm{r}=0.715)$ (48). Hence, companies and surgeons endeavor to simplify the anastomosis of the prosthesis to the distal 
aortic stump allowing less HCA time and starting rewarming swiftly (55). In addition to the HCA time, the degree of hypothermia and cerebral perfusion method to achieve the best neurologic outcome is of paramount (56). In the present series of studies included in the meta-analysis, only four authors did not mentioned the cerebral perfusion method, while in all other reports antegrade cerebral perfusion has been utilized.

\section{Limitations}

The present findings are limited by several key constraints which should be considered when interpreting the results. Firstly, all the studies were retrospective, and the main results are based on non-randomized observational studies, which impose a considerable selection bias. Secondly, due to the lack of a standard reporting schema for total arch replacement using FET, some important data are missing, which incapacitates us to perform subgroup analysis.

\section{Conclusions}

In summary, the FET procedure provides an optimal option with an acceptable rate of mortality and adverse neurologic events. As FET is gaining more popularity for aortic arch and proximal descending aorta procedures, the magnitude of operative adjuncts on the outcomes became more apparent. A lot of effort must be put into reducing HCA time and improving device profile to achieve the minimum time for graft anastomosis.

\section{Conflict of interests}

The authors have none to declare.

\section{References}

1. Borst HG, Walterbusch G, Schaps D. Extensive aortic replacement using "elephant trunk" prosthesis. Thorac Cardiovasc Surg. 1983;31(1):37-40.

2. Kato M, Ohnishi K, Kaneko M, Ueda T, Kishi D, Mizushima T, et al. New graft-implanting method for thoracic aortic aneurysm or dissection with a stented graft. Circulation. 1996;94(9 Suppl):Ii188-93.

3. Karck M, Chavan A, Hagl C, Friedrich H, Galanski M. The frozen elephant trunk technique: a new treatment for thoracic aortic aneurysms. Journal of thoracic and cardiovascular surgery. 2003;125(6):1550-3.

4. Etz CD, Plestis KA, Kari FA, Luehr M, Bodian CA, Spielvogel D, et al. Staged repair of thoracic and thoracoabdominal aortic aneurysms using the elephant trunk technique: a consecutive series of 215 first stage and 120 complete repairs. European journal of cardio-thoracic surgery. 2008;34(3):605-15.

5. Jakob H, Tsagakis K, Pacini D, Di Bartolomeo R, Mestres C, Mohr F. The International E-vita Open Registry: data sets of 274 patients. J Cardiovasc Surg (Torino). 2011;52(5):717-23.

6. Flores J, Kunihara T, Shiiya N, Yoshimoto K, Matsuzaki K, Yasuda K. Extensive deployment of the stented elephant trunk is associated with an increased risk of spinal cord injury. The Journal of thoracic and cardiovascular surgery. 2006;131(2):336-42.

7. Preventza O, Liao JL, Olive JK, Simpson K, Critsinelis AC, Price MD, et al. Neurologic complications after the frozen elephant trunk procedure: A meta-analysis of more than 3000 patients. The Journal of thoracic and cardiovascular surgery. 2020;160(1):20-33. e4.

8. Liberati A, Altman DG, Tetzlaff J, Mulrow C, Gøtzsche PC, Ioannidis JP, et al. The PRISMA statement for reporting systematic reviews and meta-analyses of studies that evaluate health care interventions: explanation and elaboration. Journal of clinical epidemiology. 2009;62(10):e1-e34.

9. Higgins JP, Thompson SG, Deeks JJ, Altman DG. Measuring inconsistency in meta-analyses. BMJ (Clinical research ed). 2003;327(7414):557-60.

10. Harbord RM, Egger M, Sterne JA. A modified test for small-study effects in meta-analyses of controlled trials with binary endpoints. Statistics in medicine. 2006;25(20):3443-57. 
11. Akbulut M, Ak A, Arslan O, Cekmecelioğlu D, Taş S, Dönmez AA, et al. Early and mid-term results of frozen elephant trunk procedure for acute type A aortic dissection. Turkish Journal of Thoracic and Cardiovascular Surgery. 2019;27(2):135.

12. Berger T, Kreibich M, Morlock J, Kondov S, Scheumann J, Kari FA, et al. True-lumen and false-lumen diameter changes in the downstream aorta after frozen elephant trunk implantation. European Journal of Cardio-Thoracic Surgery. 2018;54(2):375-81.

13. Chen I, Chen P-l, Weng S-h, Hsu C-p, Shih C-c, Chang H-h, et al. Clinical Outcomes of VasoRing Connector in Patients With Acute Type A Aortic. 2018.

14. Chen L-W, Lu L, Dai X-F, Wu X-J, Zhang G-C, Yang G-F, et al. Total arch repair with open triplebranched stent graft placement for acute type A aortic dissection: experience with 122 patients. The Journal of thoracic and cardiovascular surgery. 2014;148(2):521-8.

15. Chen Q, Cheng F, Chen T, Zhao F, Jiang N. Ascending aorta replacement combined with open placement of triple-branched stent graft and total arch replacement combined with stented elephant trunk implantation for treating type A aortic dissection. Int J Clin Exp Med. 2016;9(8):16070-7.

16. Furutachi A, Takamatsu M, Nogami E, Hamada K, Yunoki J, Itoh M, et al. Early and mid-term outcomes of total arch replacement with the frozen elephant trunk technique for type A acute aortic dissection. Interactive cardiovascular and thoracic surgery. 2019;29(5):753-60.

17. Goebel N, Nagib R, Salehi-Gilani S, Ahad S, Albert M, Ursulescu A, et al. One-stage hybrid aortic repair using the frozen elephant trunk in acute DeBakey type I aortic dissection. Journal of thoracic disease. 2018;10(7):4195.

18. Gong M, Wu Z, Xu S, Li L, Wang X, Guan X, et al. Increased risk for the development of postoperative severe hypoxemia in obese women with acute type a aortic dissection. Journal of cardiothoracic surgery. 2019;14(1):1-9.

19. Guan X, Gong M, Wang X, Zhu J, Liu Y, Sun L, et al. Low preoperative fibrinogen level is risk factor for neurological complications in acute aortic dissection. Medicine. 2018;97(21).

20. Guan XL, Wang XL, Liu YY, Lan F, Gong M, Li HY, et al. Changes in the hemostatic system of patients with acute aortic dissection undergoing aortic arch surgery. The Annals of thoracic surgery. 2016;101(3):94551.

21. He J, Peng J, Li W, Zheng D, Cai S, Xu W, et al. "Aorta-clamp" technique for surgical repair of acute type A aortic dissection - 5 min circulatory arrest at $30^{\circ}$ C. Journal of thoracic disease. 2019;11(11):4717.

22. Hoffman A, Damberg AL, Schälte G, Mahnken AH, Raweh A, Autschbach R. Thoracic stent graft sizing for frozen elephant trunk repair in acute type A dissection. The Journal of thoracic and cardiovascular surgery. 2013;145(4):964-9. e1.

23. Hohri Y, Yamasaki T, Matsuzaki Y, Hiramatsu T. Early and mid-term outcome of frozen elephant trunk using spinal cord protective perfusion strategy for acute type A aortic dissection. General thoracic and cardiovascular surgery. 2020:1-9.

24. Jiang H, Liu Y, Yang Z, Ge Y, Li L, Wang H. Total arch replacement via single upper-hemisternotomy approach in patients with type A dissection. The Annals of thoracic surgery. 2020;109(5):1394-9.

25. Katayama A, Uchida N, Katayama K, Arakawa M, Sueda T. The frozen elephant trunk technique for acute type A aortic dissection: results from 15 years of experience. European Journal of Cardio-Thoracic Surgery. 2015;47(2):355-60.

26. Kobayashi M, Chaykovska L, van der Loo B, Nguyen TDL, Puippe G, Salzberg S, et al. Long-term results of simplified frozen elephant trunk technique in complicated acute type A aortic dissection: A case-control study. Vascular. 2016;24(5):523-30. 
27. Koechlin L, Kaufmann J, Macius E, Bremerich J, Sommer G, Gahl B, et al. Impact of Modified Frozen Elephant Trunk Procedure on Downstream Aorta Remodeling in Acute Aortic Dissection: CT Scan FollowUp. World journal of surgery. 2020;44(5):1648-57.

28. Leontyev S, Tsagakis K, Pacini D, Di Bartolomeo R, Mohr FW, Weiss G, et al. Impact of clinical factors and surgical techniques on early outcome of patients treated with frozen elephant trunk technique by using EVITA open stent-graft: results of a multicentre study. Oxford University Press; 2016.

29. Li J, Sun Y, Zhou T, Wang Y, Sun Y, Lai H, et al. David reimplantation with simultaneous total arch replacement and stented elephant trunk for acute type A aortic dissection. The Journal of thoracic and cardiovascular surgery. 2019.

30. Ma M, Feng X, Wang J, Dong Y, Chen T, Liu L, et al. Acute Type I aortic dissection: a propensitymatched comparison of elephant trunk and arch debranching repairs. Interactive cardiovascular and thoracic surgery. 2018;26(2):183-9.

31. Ma M, Liu L, Feng X, Wang Y, Hu M, Pan T, et al. Moderate hypothermic circulatory arrest with antegrade cerebral perfusion for rapid total arch replacement in acute type A aortic dissection. The Thoracic and cardiovascular surgeon. 2016;64(02):124-32.

32. Ma W-G, Zheng J, Zhang W, Sun K, Ziganshin BA, Wang L-F, et al. Frozen elephant trunk with total arch replacement for type A aortic dissections: does acuity affect operative mortality? The Journal of thoracic and cardiovascular surgery. 2014;148(3):963-72.

33. Roselli EE, Idrees JJ, Bakaeen FG, Tong MZ, Soltesz EG, Mick S, et al. Evolution of simplified frozen elephant trunk repair for acute DeBakey type I dissection: midterm outcomes. The Annals of thoracic surgery. 2018;105(3):749-55.

34. Shen K, Tang H, Jing R, Liu F, Zhou X. Application of triple-branched stent graft for Stanford type A aortic dissection: potential risks. European Journal of Cardio-Thoracic Surgery. 2012;41(3):e12-e7.

35. Shi E, Gu T, Yu Y, Wang C, Yu L, Fang Q, et al. Simplified total arch repair with a stented graft for acute DeBakey type I dissection. The Journal of thoracic and cardiovascular surgery. 2014;148(5):2147-54.

36. Shrestha M, Bachet J, Bavaria J, Carrel TP, De Paulis R, Di Bartolomeo R, et al. Current status and recommendations for use of the frozen elephant trunk technique: a position paper by the Vascular Domain of EACTS. European Journal of Cardio-Thoracic Surgery. 2015;47(5):759-69.

37. sUNL Q. Totalarchreplacementcombinedwith stentedelephanttrunkimplantation: anew" standard" therapyfor typeadissectioninvolvingrepairoftheaorticarch? Circulation. 2011;123(9):971.

38. Tan L, Xiao J, Zhou X, Shen K, Li F, Luo J, et al. Untreated distal intimal tears may be associated with paraplegia after total arch replacement and frozen elephant trunk treatment of acute Stanford type A aortic dissection. The Journal of thoracic and cardiovascular surgery. 2019;158(2):343-50. e1.

39. Xiao Z, Meng W, Zhu D, Guo Y, Zhang E. Treatment strategies for left subclavian artery during total arch replacement combined with stented elephant trunk implantation. The Journal of thoracic and cardiovascular surgery. 2014;147(2):639-43.

40. Yamamoto H, Kadohama T, Yamaura G, Tanaka F, Takagi D, Kiryu K, et al. Total arch repair with frozen elephant trunk using the "zone 0 arch repair" strategy for type A acute aortic dissection. The Journal of thoracic and cardiovascular surgery. 2020;159(1):36-45.

41. Yang S-M, Xu P, Li C-X, Huang Q, Gao H-B, Li Z-F, et al. A modified total arch replacement combined with a stented elephant trunk implantation for acute type A dissection under deep hypothermic circulatory arrest and selective antegrade cerebral perfusion. Journal of cardiothoracic surgery. 2014;9(1):1-6.

42. Yoshitake A, Tochii M, Tokunaga C, Hayashi J, Takazawa A, Yamashita K, et al. Early and long-term results of total arch replacement with the frozen elephant trunk technique for acute type A aortic dissection. 
European Journal of Cardio-Thoracic Surgery. 2020;58(4):707-13.

43. Yu B, Liu Z, Xue C, Liu J, Yang J, Jin Z, et al. Total arch repair with open placement of a novel doublebranched stent graft for acute Type A aortic dissection: a single-centre experience with 21 consecutive patients. Interactive cardiovascular and thoracic surgery. 2019;28(2):262-9.

44. Zhang H, Lang X, Lu F, Song Z, Wang J, Han L, et al. Acute type A dissection without intimal tear in arch: proximal or extensive repair? The Journal of thoracic and cardiovascular surgery. 2014;147(4):1251-5.

45. Moulakakis KG, Mylonas SN, Markatis F, Kotsis T, Kakisis J, Liapis CD. A systematic review and meta-analysis of hybrid aortic arch replacement. Annals of cardiothoracic surgery. 2013;2(3):247.

46. Rezaei Y, Bashir M, Mousavizadeh M, Daliri M, Aljadayel HA, Mohammed I, et al. Frozen elephant trunk in total arch replacement: A systematic review and meta-analysis of outcomes and aortic proximalization. Journal of Cardiac Surgery. 2021.

47. Hanif H, Dubois L, Ouzounian M, Peterson MD, El-Hamamsy I, Dagenais F, et al. Aortic arch reconstructive surgery with conventional techniques vs frozen elephant trunk: a systematic review and meta-analysis. Canadian Journal of Cardiology. 2018;34(3):262-73.

48. Tian DH, Wan B, Di Eusanio M, Black D, Yan TD. A systematic review and meta-analysis on the safety and efficacy of the frozen elephant trunk technique in aortic arch surgery. Annals of cardiothoracic surgery. $2013 ; 2(5): 581$.

49. Czerny M, Schmidli J, Adler S, Van Den Berg JC, Bertoglio L, Carrel T, et al. Current options and recommendations for the treatment of thoracic aortic pathologies involving the aortic arch: an expert consensus document of the European Association for Cardio-Thoracic surgery (EACTS) and the European Society for Vascular Surgery (ESVS). European journal of cardio-thoracic surgery. 2019;55(1):133-62.

50. Hagl C, Ergin MA, Galla JD, Lansman SL, McCullough JN, Spielvogel D, et al. Neurologic outcome after ascending aorta-aortic arch operations: effect of brain protection technique in high-risk patients. The Journal of thoracic and cardiovascular surgery. 2001;121(6):1107-21.

51. Miyairi T, Kotsuka Y, Ezure M, Ono M, Morota T, Kubota H, et al. Open stent-grafting for aortic arch aneurysm is associated with increased risk of paraplegia. The Annals of thoracic surgery. 2002;74(1):83-9.

52. Mizuno T, Toyama M, Tabuchi N, Wu H, Sunamori M. Stented elephant trunk procedure combined with ascending aorta and arch replacement for acute type A aortic dissection. European journal of cardio-thoracic surgery. 2002;22(4):504-9.

53. Etz CD, Kari FA, Mueller CS, Brenner RM, Lin H-M, Griepp RB. The collateral network concept: remodeling of the arterial collateral network after experimental segmental artery sacrifice. The Journal of thoracic and cardiovascular surgery. 2011;141(4):1029-36.

54. De Paulis R, Czerny M, Weltert L, Bavaria J, Borger MA, Carrel TP, et al. Current trends in cannulation and neuroprotection during surgery of the aortic arch in Europe. European journal of cardio-thoracic surgery. 2015;47(5):917-23.

55. Ius F, Fleissner F, Pichlmaier M, Karck M, Martens A, Haverich A, et al. Total aortic arch replacement with the frozen elephant trunk technique: 10-year follow-up single-centre experience + . European Journal of Cardio-Thoracic Surgery. 2013;44(5):949-57.

56. Suarez FEF, Del Valle DF, Alvarez AG, Perez-Lozano B. Intraoperative care for aortic surgery using circulatory arrest. Journal of thoracic disease. 2017;9(Suppl 6):S508.

Figure legends

Figure 1- PRISMA flow diagram

Figure 2- Pooled estimates of (A) postoperative stroke and (B) SCI 
Figure 3- Pooled estimates of (A) acute kidney injury, (B) bleeding, and (C) in-hospital mortality

Figure 4- The graph shows the effect sizes for (A) postoperative stroke and (B) SCI plotted against the HCA time. The regression line and corresponding confidence intervals are plotted

Supplementary Figure 1- Funnel plots showing the publication bias for (A) postoperative stroke, (B) SCI, (C) acute kidney injury, (D) bleeding, and (E) in-hospital mortality

\section{Hosted file}

Tables.pdf available at https://authorea.com/users/340498/articles/519787-hypothermiccirculatory-arrest-time-affects-neurological-outcomes-of-frozen-elephant-trunk-foracute-type-a-aortic-dissection-a-systematic-review-and-meta-analysis

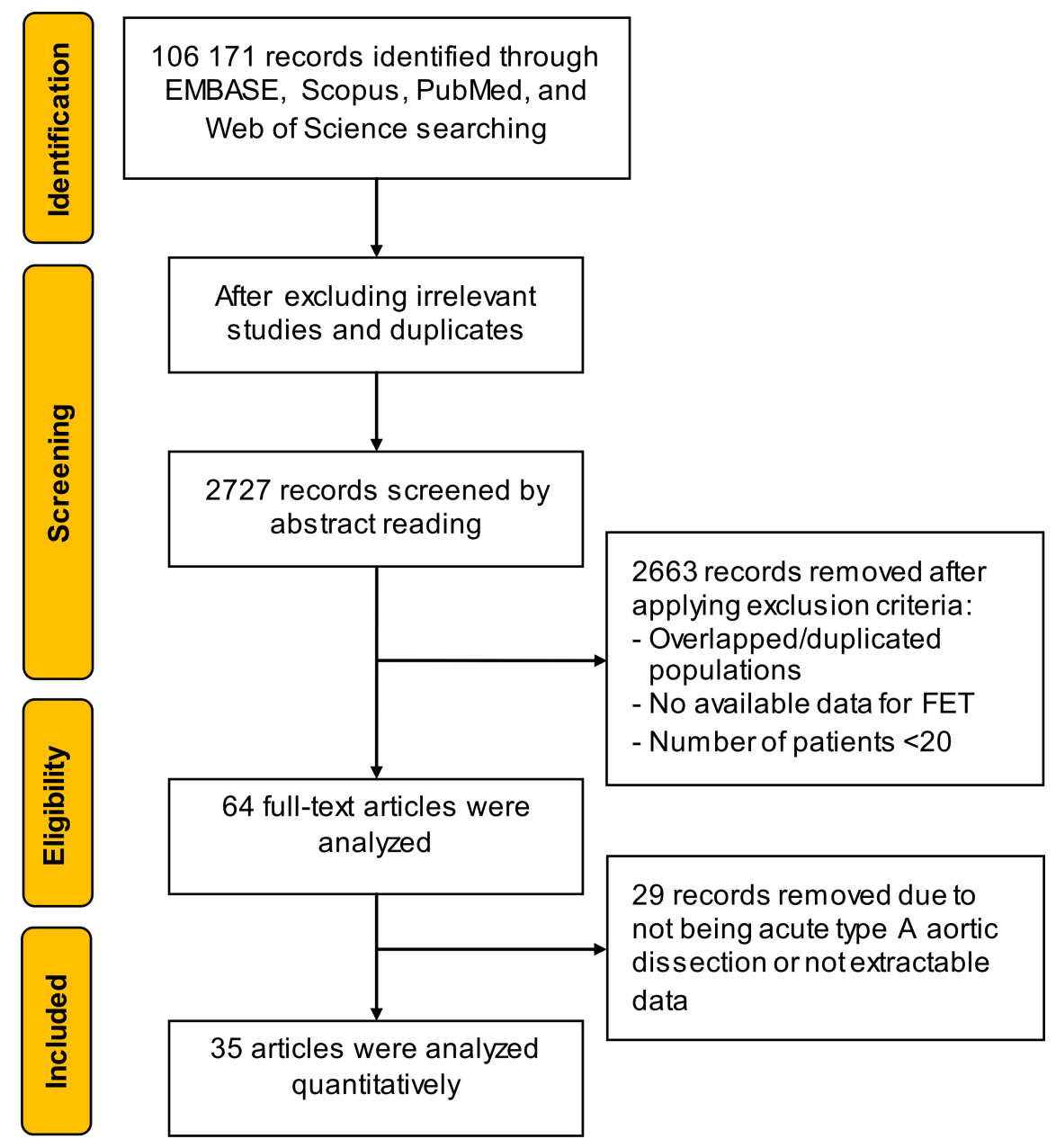




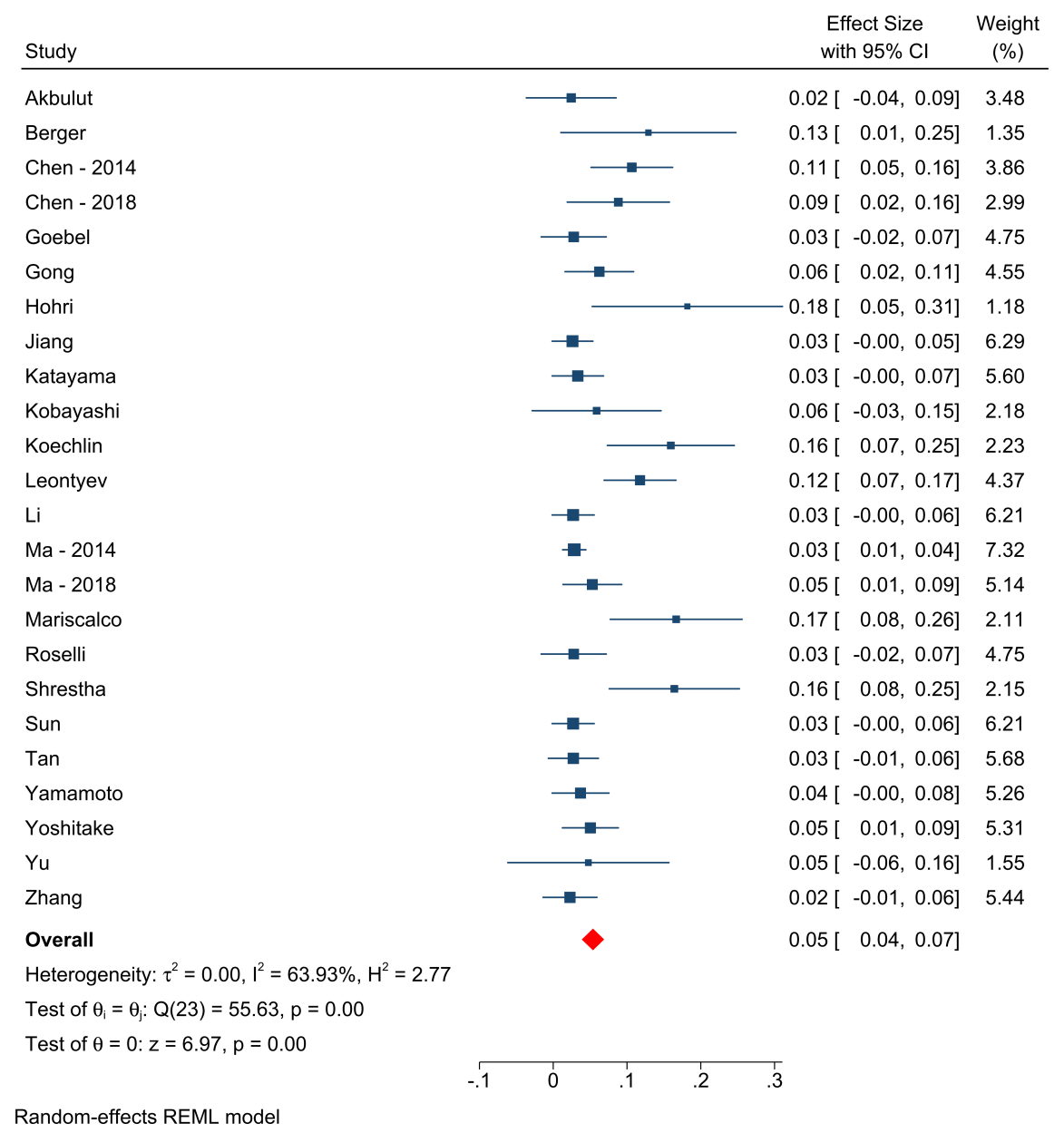




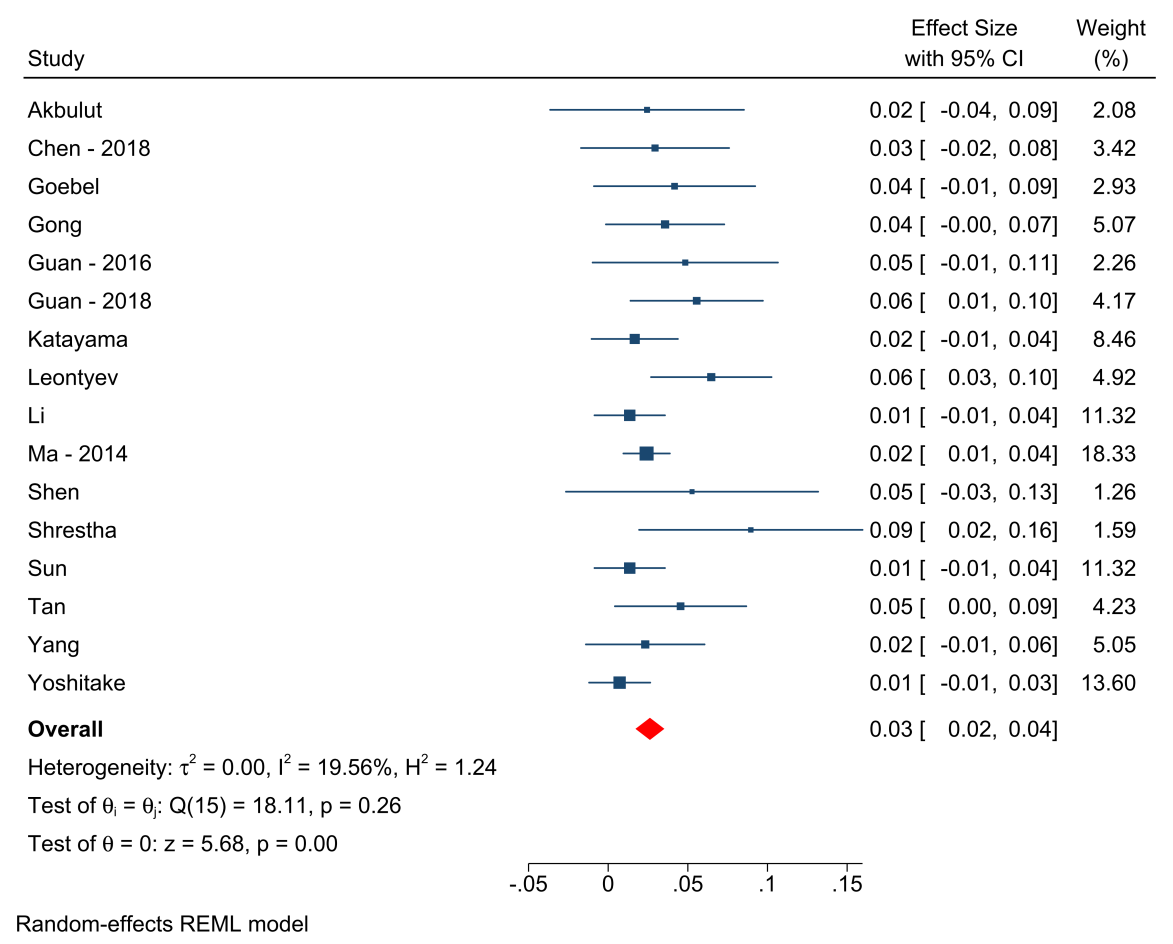




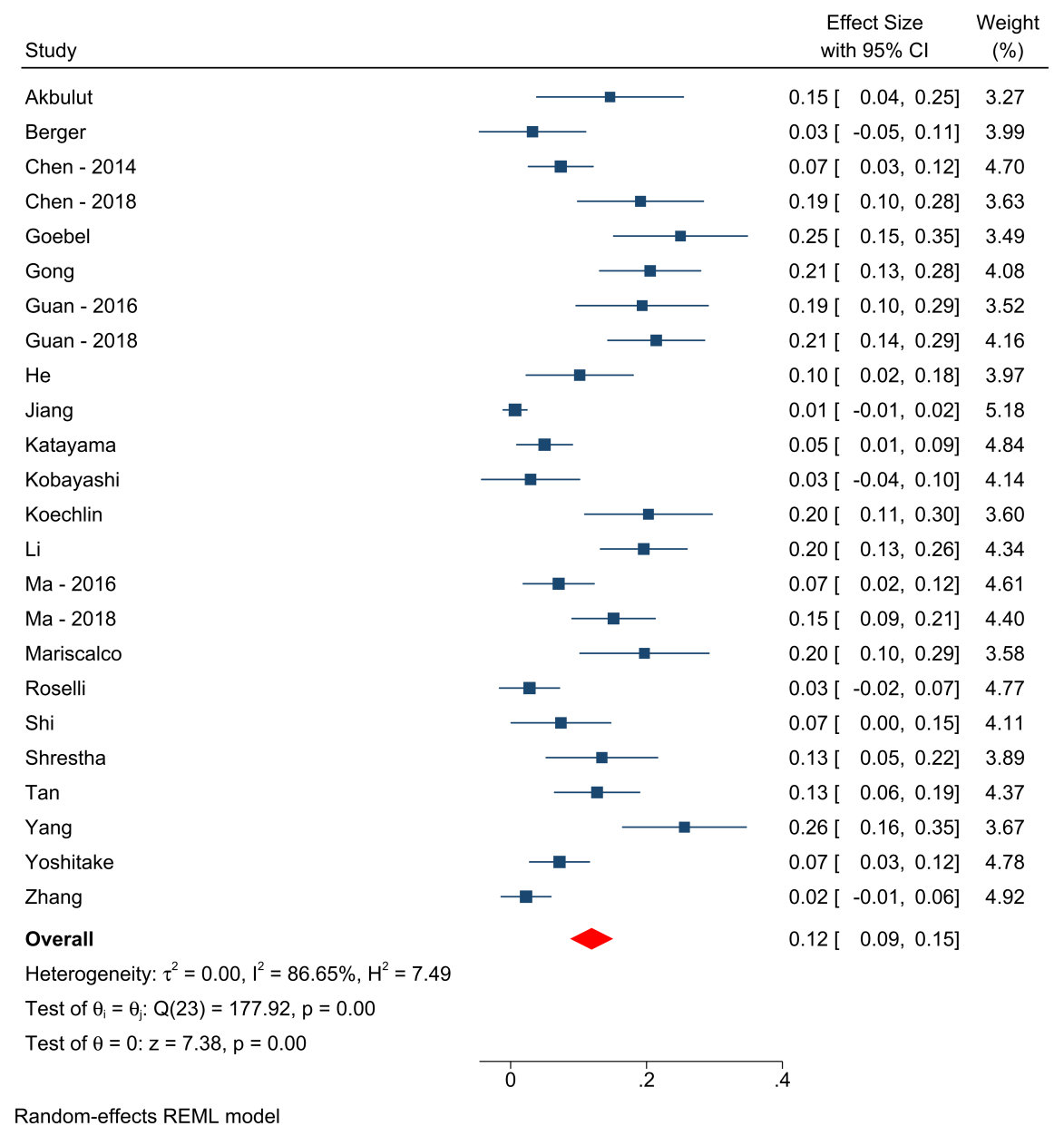

Effect Size Weight

Study $\quad$ with $95 \% \mathrm{Cl} \quad(\%)$

Hoffman $\quad \ldots \quad 0.13$ [ $0.01,0.24] \quad 4.61$

Koechlin

Berger

Katayama

Shrestha

Yang

Guan - 2018

Jiang

Li

Overall

Heterogeneity: $\tau^{2}=0.00, \mathrm{I}^{2}=71.05 \%, \mathrm{H}^{2}=3.45$

Test of $\theta_{i}=\theta_{j}: Q(8)=26.11, p=0.00$

Test of $\theta=0: z=3.79, p=0.00$ $0.04[-0.01,0.10] 11.52$ $0.06[-0.03,0.16] \quad 6.14$ $0.03[-0.00,0.07] 14.81$ $0.18[0.09,0.27] \quad 6.47$ $0.08[0.02,0.14] 10.42$ 0.07 [ $0.03,0.12] 12.69$ $0.03[0.00,0.06] 15.68$ $0.01[-0.01,0.02] 17.65$ 0.06 [ $0.03,0.08$ ]

Random-effects REML model

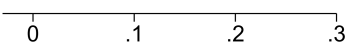




\begin{tabular}{|c|c|c|c|}
\hline Study & & $\begin{array}{c}\text { Effect Size } \\
\text { with } 95 \% \mathrm{Cl}\end{array}$ & $\begin{array}{c}\text { Weight } \\
(\%)\end{array}$ \\
\hline Akbulut & $\longrightarrow$ & $0.15[0.04,0.25]$ & 2.14 \\
\hline Berger & $\longrightarrow$ & $0.06[-0.03,0.16]$ & 2.56 \\
\hline Chen - 2016 & 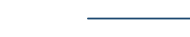 & $-0.27[0.10,0.45]$ & 1.00 \\
\hline Gong & $\rightarrow-$ & $0.05[0.01,0.10]$ & 5.21 \\
\hline Guan - 2016 & $\longrightarrow$ & $0.10[0.02,0.17]$ & 3.36 \\
\hline Guan - 2018 & $\longrightarrow$ & $0.18[0.12,0.25]$ & 3.77 \\
\hline $\mathrm{He}$ & $\longrightarrow$ & $0.03[-0.02,0.09]$ & 4.60 \\
\hline Jiang & $\rightarrow-$ & $0.05[0.02,0.09]$ & 5.69 \\
\hline Katayama & - & $0.02[-0.01,0.04]$ & 6.30 \\
\hline Koechlin & $\longrightarrow$ & $0.07[0.01,0.14]$ & 3.96 \\
\hline Leontyev & $\longrightarrow$ & $0.17[0.11,0.23]$ & 4.39 \\
\hline $\mathrm{Li}$ & $\rightarrow-$ & $0.07[0.03,0.11]$ & 5.36 \\
\hline Ma - 2014 & $\rightarrow$ & $0.08[0.06,0.11]$ & 6.41 \\
\hline Mariscalco & $\longrightarrow$ & $0.12[0.04,0.20]$ & 3.17 \\
\hline Roselli & $\longrightarrow$ & $0.04[-0.01,0.09]$ & 4.75 \\
\hline Shen & $\longrightarrow$ & $0.08[-0.01,0.17]$ & 2.71 \\
\hline Shi & $\longrightarrow$ & $0.04[-0.02,0.09]$ & 4.31 \\
\hline Sun & $\rightarrow-$ & $0.05[0.01,0.08]$ & 5.75 \\
\hline Xiao & 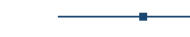 & $0.18[0.05,0.31]$ & 1.65 \\
\hline Yamamoto & $\longrightarrow$ & $0.06[0.02,0.11]$ & 4.92 \\
\hline Yang & $\longrightarrow$ & $0.06[0.01,0.11]$ & 4.67 \\
\hline Yoshitake & $\rightarrow$ & $0.01[-0.01,0.04]$ & 6.51 \\
\hline $\mathrm{Yu}$ & $\rightleftarrows$ & $0.05[-0.06,0.16]$ & 2.11 \\
\hline Zhang & $\rightarrow-$ & $0.06[0.01,0.11]$ & 4.74 \\
\hline Overall & $>$ & $0.07[0.05,0.09]$ & \\
\hline \multicolumn{4}{|c|}{ Heterogeneity: $\tau^{2}=0.00, \mathrm{I}^{2}=68.65 \%, \mathrm{H}^{2}=3.19$} \\
\hline \multicolumn{4}{|c|}{ Test of $\theta_{i}=\theta_{j}: Q(23)=72.14, p=0.00$} \\
\hline \multicolumn{4}{|c|}{ Test of $\theta=0: z=7.40, p=0.00$} \\
\hline & .2 & & \\
\hline
\end{tabular}

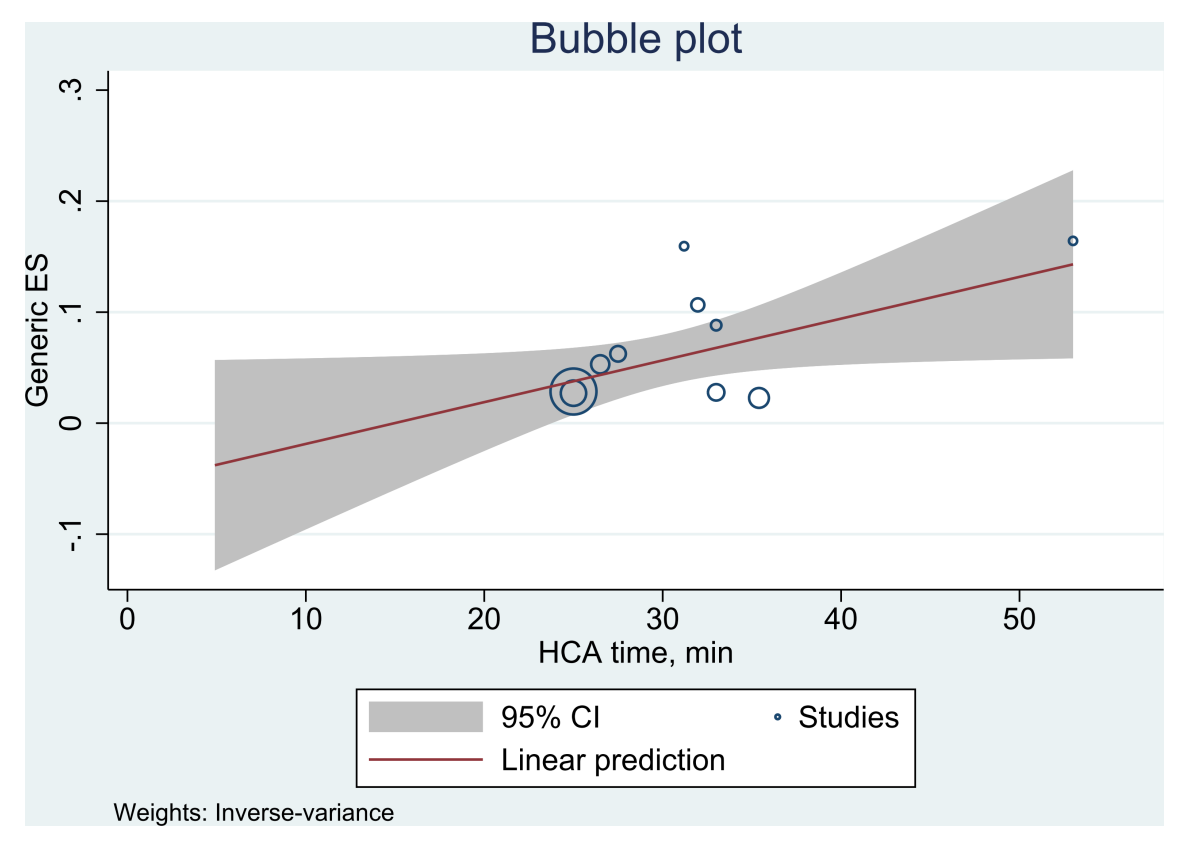




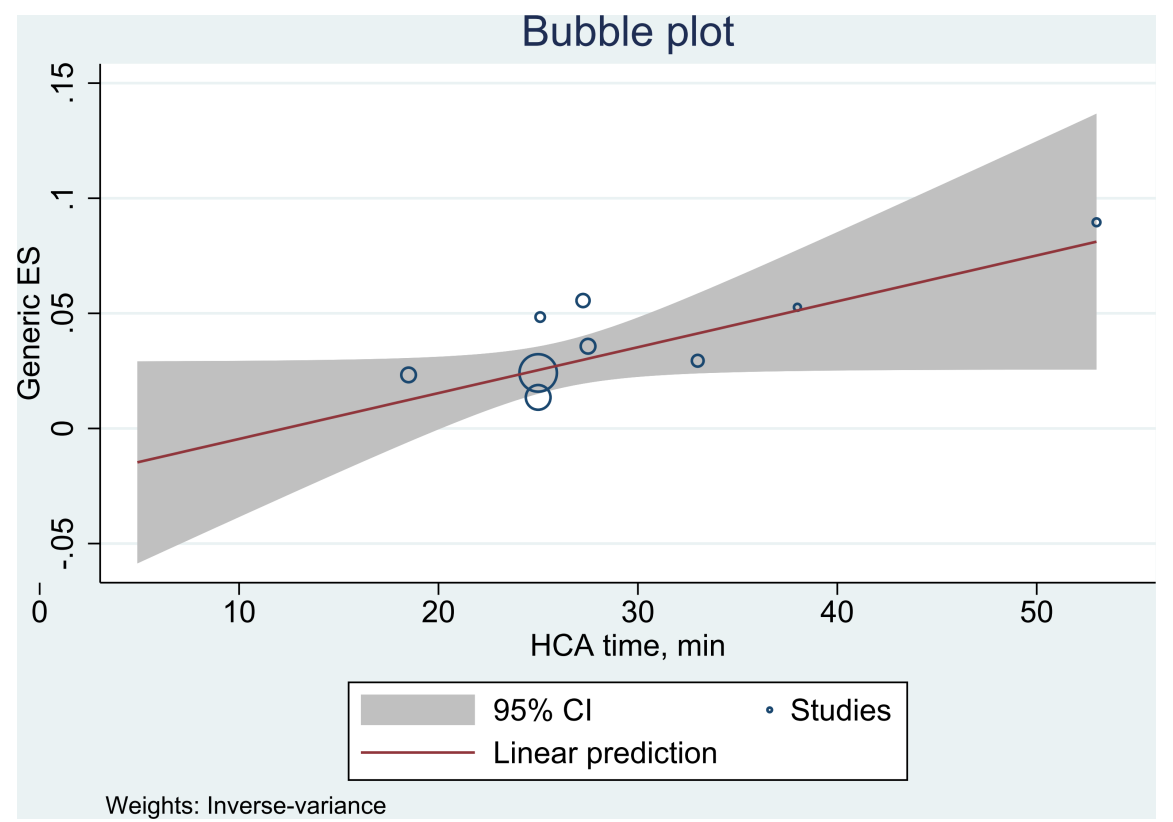

